mended very highly. Shortly after the meeting 1 had occasion to dress a contused wound of the hand with it. The patient was a workman in a blast furnace. I washed the wound carefully at first in order to remove all of the septic matter, and I thought $I$ had gotten it all out of the wound. I then sprinkled the acetanilid over it according to the directions given by the gentleman referred to. The result in that case was the development of an erysipelatous inflammation. I converted the wound into an ugly condition, so that it took the patient three or four times as long to get well as it would have done if the wound had been dressed with gauze, iodoform, or subcarbonate of bismuth. The latter has no odor and is as good as iodoform in its results. After adopting the method suggestad by this gentleman I was discouraged, and I have not since applied it on a wound. I have tried it on small wounds, and I find that it acts as an irritant.

A Member--.Was the wound very much contused?

DR. Griswold-It was not very much contused. It was a lacerated wound on the back of the hand and not a very large one, but an erysipelatous inflammation developed over the back of the hand as a result of the use of the acetanilid. After using it several times subsequently in smaller wounds, I thought the doctor who recommended it had made a mistake.

Dr. REger (closing the discussion)-I believe there is no difference between myself and my critics. I have not for several years applied antiseptics to the inside of a wound, as the paper alleges. A man is brought to you, we will say, with his limbs crushed and covered with cinders, dirt and grease, and the only place where I would use an antiseptic is in preparing the part for amputation. In crushed wounds you will always find to a greater or less distance from the wound that the tissues are entirely devitalized, and consequently with a few exceptions the surgeon can make his amputation through sound tissue. I do not irrigate. I use sterilized gauze sponges and if there is any oozing of blood after thesurface is closed it must be aseptic and must absorb harmlessly. I use all my antiseptics prior to the operation. I take everything along with me to make my operation as nearly aseptic as I can. The sheet is for the purpose of preventing any particle from falling into the wound.

\section{VAGINAL LIGATION OF THE BROAD LIGA- MENTS FOR FIBROIDS OF THE UTERUS AND OTHER MINOR SURGICAL TREATMENT.}

BY FRANKLIN H. MARTIN, M.D.

PROFESSOR OF GYNFCOLOGY POST-GRADUATE MEDICAL SCHOOL, SURGEON TO WOMAN'S HOSPITAL. CIICAGO.

Vaginal ligation of the contents of the base of the broad ligaments, for the cure of fibroids of the uterus, was devised and performed by me as a new and original operation Nov. 15, 1892, and was described and published in the April number of the American Journal of Olsstetive in 1893 . In the January number of the Americen. Journal of Obstetrics, 1894, I reported six cases treated by the new operation.

The operation as originally described by me is as follows: The ligation of more or less of the broad ligament of the uterus, with its vessels and nerves, the extent of the ligation depending upon the result sought, from a simple ligation of the base of the ligament, including the uterine arteries and branches of both sides without opening the peritoneum to a complete ligation of the ligament of one side, including both uterine and ovarian arteries, with partial ligation of the opposite ligament without opening the peritoneal cavity, if possible, but by doing so if necessary.
The results sought in the operation are, first to check uterine hemorrhages by cutting off blood channels, and secondly to produce atrophy of the fibroid by, 1 , depriving it of nourishment through the blood vessels and, 2, by changing the nutrition of the uterus by interfering with its nerve supply.

Immediately after publishing my first article on this operation there were two claimants for priority; Dr. Walter B. Dorsett, of St. Louis, and Prof. S. Gottschalk, of Berlin, Germany. Dr. Dorsett, in a letter to the American Journal of Obstetrics, claimed that he had suggested a similar procedure to my operation in an article he published in the St. Louis Courier of Merlicine in 1890 , the article bearing title of "A Case of Atrophy of the Female Genitalia following Pregnancy and remarks." In this article he made the following observation: "I believe that in the treatment of uterine fibroid . . . to ligate the uterine artery would not be an unscientific procedure. On the contrary the more I have thought of it the more I am inclined to believe that it would be the most certain mode of treatment." Dr. Dorsett, while advancing the theory, had not at that time carried it out on a living woman.

Prof. Gottschalk based his claim of priority on an article read by him at the Brussels Congress, Sept. 16,1892 , with the following title: "Die Histogenese und Aetiologie der Uterusmyome." In the latter paragraphs of this article he casually suggested ligation of the uterine arteries and stated that he had performed the operation twice. This is what he said: "The bilateral ligation of the uterine arteries appears to be the therapeutic measure in this regard for the earliest incipient stages of myoma. This offers no difficulties in its technique; it is easily performed in a few minutes. . I I have already performed this ligation in two cases in which I was able to early diagnose the development of multiple myoma with best results."

Thus these two men both suggested tying the uterine arteries for the cure of fibroids and at least one of them (Gottschalk) performed the operation twice before I described my operation. This would definitely decide the question of priority in their favor if the operation they suggested was identical with mine. Their operation is not identical in theory, in execution, or in description with mine, and therefore their claim of priority for my operation can not be substantiated.

The operation suggested by these men simply includes the ligating of the uterine artery from the vagina, while, 1 , I ligate in all cases, the whole base of the broad liyament, in order, a, to occlude not only the main channel of the uterine artery, but all collateral branches; $b$, in order to destroy the function of the nerves as well as the arteries of nutrition; $c$, in order to diminish nerve reflexes. 2. I include, in desperate cases, not only the base of the broad ligament with the uterine artery and branches in my ligatures, but when practicable ligate high enough on one side to take in the ovarian artery. 3. I advise accomplishing this result, if possible, without opening the peritoneal cavity, but by doing so, if necessary.

\section{TECHNIQUE OF OPERATION.}

The preparation of a patient for vaginal ligation of the broad ligaments of the uterus should be similar to that demanded for vaginal hysterectomy, as 
described in my article last week. Ether is used as an anesthetic and the patient is placed on the operating table in the exaggerated lithotomy position with buttocks brought to end of the table, with an assistant on either side to support the limbs and hold the vaginal retractors. A broad, short vaginal retractor above and below exposes the cervix, which is transfixed with a strong silk ligature to be employed in handling the uterus. The uterine canal is dilated and the uterine cavity curetted with a dull curette and thoroughly irrigated with 1:1000 bichlorid solution and then loosely packed with iodoform gauze. This procedure cleans the uterus and makes it impossible for the vaginal wounds to become infected by a septic uterine discharge. The uterus is now drawn down in order to put the broad ligaments on the stretch and then drawn to the right side so as to expose the left vaginal vault. The mucous membrane of the vagina at the utero-vaginal fold on the left side is then caught with a tenaculum and incised with a pair of curved scissors. One blade is allowed to enter beneath

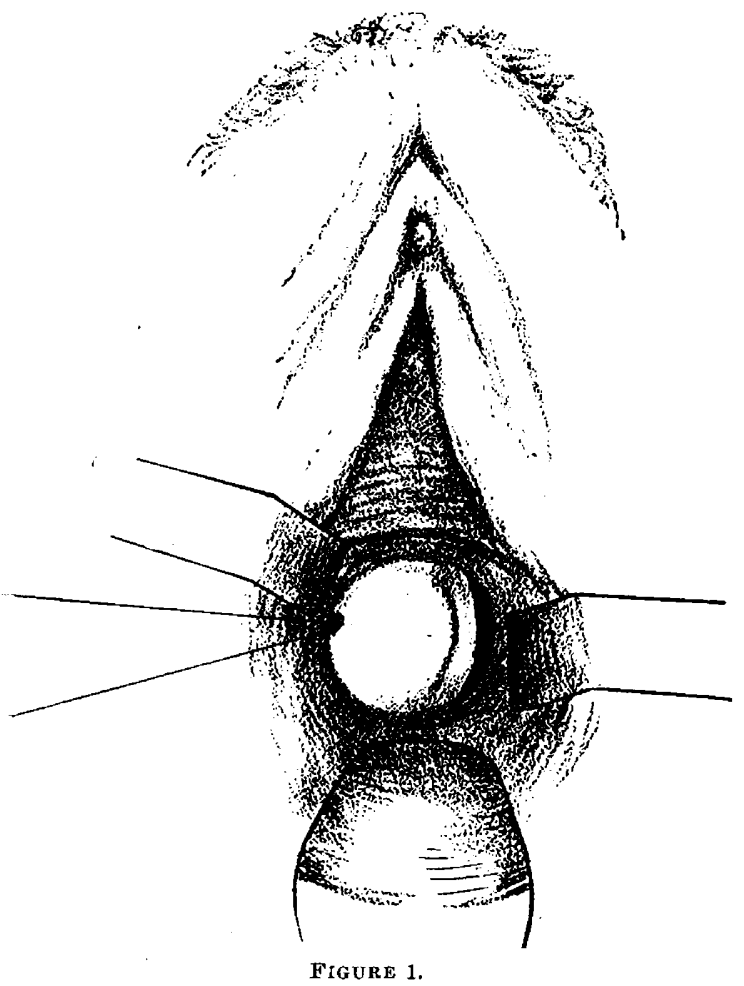

the mucous membrane and a curved incision one and one-half to two inches long is made over the broad ligament and at right angles to it (Fig. 1). By means of the index fingers of the two hands the operator now separates the vaginal tissue from the broad ligament and carefully separates the broad ligament in front from the bladder for a height of two inches and laterally for nearly the same distance (Fig. 2). The bladder should be carefully separated in this way in order to avoid the danger of wounding the organ, and by pushing the separation laterally the ureter is forced out of danger. One then carefully separates the broad ligament posteriorly to the same height as in front, without, if possible, penetrating the peritoneum. Now, by passing one finger behind the other in front, the whole base of the broad ligament, representing twothirds of its bulk, can be grasped (Fig. 3) for a distance of an inch to an inch and a half from the uterus. In uterus, and these represent 75 per cent. of all fibroids this grasp one can easily feel the throb of the main of the uterus, wherever it is possible to tie the base of 
the broad ligament from the vagina, this operation/She has a slight menstrual flow each month, and is may be expected to accomplish prompt and decided free from pain.

relief of symptoms and a rapid reduction of the tumor. The cases in which the most satisfactory results must be expected are incipient or small fibroids of the interstitial variety which show themselves late in the menstrual life. Here, we have a uterus which is small enough so that it has not risen above the brim of the pelvis, one which can be easily reached from the vagina so that its broad ligaments are accessible from below. Such a fibroid, too, from the age of the patient will reach a state of quiescence as soon as the menopause is established. In such cases, then, a major operation is particularly undesirable, because it is not imperatively demanded and because of a reasonable chance of relief at the approaching change; on the other hand the symptoms (with severe hemorrhage usually as the principal one) are such that immediate relief is earnestly sought, if one can be reasonably certain of obtaining it without submitting to a dangerous and radical procedure. These are ideal cases for this operation.

Another class of cases in which this operation has been employed with gratifying success and in which it will probably find favor with the most radical operators, are those of continuous and profuse hemorrhage in which the desperateness of the drain is such

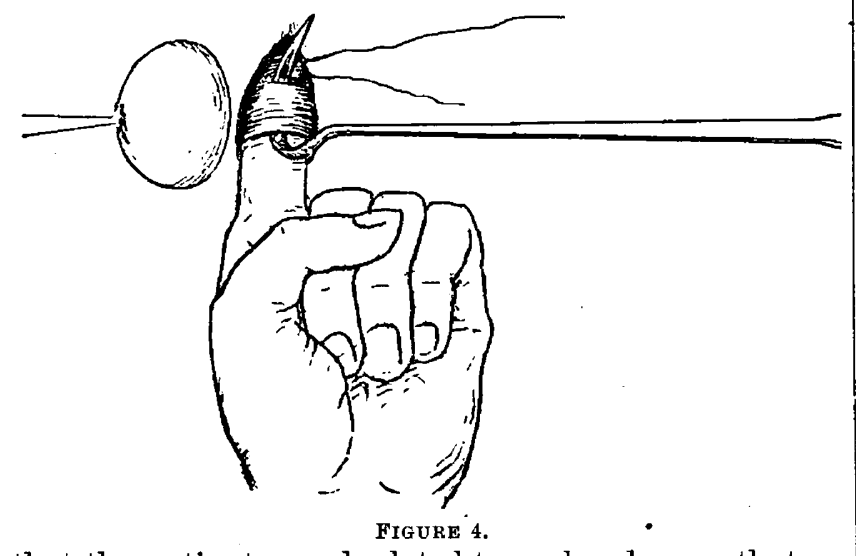

that the patients are depleted to such a degree, that no radical procedure can be thought of, until a minor operative procedure has checked blood waste and recuperation is accomplished. My fourth and sixth cases were like the above. In the fourth case hemorrhage was very profuse and the patient was completely exsanguinated and so weak that she had not been out of bed for several months. Some time before I determined to submit her to my operation an attempt had been made to remove the appendages, or, if possible, when the laparotomy was in progress, the uterus. From complicated adhesions and the weakness of the subject neither operation was possible after the abdomen had been opened. The tumor was large and the elevation of the uterus in consequence was great, and it was with the utmost difficulty with the aid of the most competent assistants, that I finally succeeded in ligating thoroughly the base of each broad ligament. Both ligaments contained several arteries, some of them as large as the normal radial artery. They were all tied in mass. Hemorrhage stopped from the instant of tying the last ligature and it has never recurred. It has now been over three years since I operated on this case. The uterus has reduced until it is but slightly larger than normal. The woman (I examined her but a few months ago) is perfectly well.

Case six was of a severe hemorrhagic nature in a typical interstitial fibroid of three by five inches in diameter. The woman was too weak and depleted for a radical operation. I did my operation on her and the result was marvelous. In three months' time she had recuperated so that any radical operation might have been done without danger.

Dr. Humiston, of Cleveland, reported to me a case in which he used my operation as a procedure of last resort, in a patient nearly moribund from hemorrhage. She was so weak that he only attempted ligation on one side. The woman stopped bleeding instantly and eventually recovered. Hence, the operation may with propriety be employed as a rational temporary expedient in desperate cases of whatever variety, where uterine blood loss is conspicuous.

\section{CASES.}

In selecting cases for this operation I have been very careful. In the majority of them I have operated on, there seemed no alternative. All were desperate ones, like cases $1,2,3,6$ and 8 , or they would not submit to a more radical procedure, and milder means, as electricity, ergot, etc., would not accomplish satisfactory results. I have been more conservative in adopting the operation, I am afraid, than the results in the few cases I have operated on would justify. One reason for not adopting the operation in a larger number of cases is that I wished first to learn of the remote results. It is now over three years since my first operation and most of the operations which I have performed were during the first year. I have,

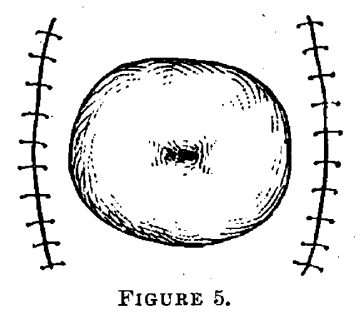

therefore, a three years' history to analyze in the majority of my cases. In the following report there are no instances in which, at least, a year has not elapsed since the operation.

Case 1.-This was an interstitial fibroid in a maiden lady 36 years old, in size extending above the umbilicus. The hemorrhage was exhaustive and the patient greatly reduced in consequence. Her heart was hypertrophied and her condition was such that no surgeon with a proper care for his statistics or his patient's life would have ventured a hysterectomy. She was operated on by my operation Nov. 15, 1892. The hemorrhage decreased about onehalf for several months after the operation. The tumor in the first four months materially decreased in size. In May, 1894, the hemorrhage is reported much modified, and no longer a source of alarm. The patient at that date considered' her condition greatly improved, hemorrhage cured, tumor materially reduced and pressure symptoms subsided. March: 12,1896 , four years and three months after the operation the patient reports herself well. The original fullness produced. by the tumor she can no longer feel. No pain. The last flowing of any consequence was November, 1894. Since then the flow has been very slight until last July, when it practically ceased. "I have color in my lips and cheeks. I walk two miles or more every day," she writes. This report is certainly very gratifying.

Case 2.-The second case was a married woman 40 years of age who had been under electrical treatment for a hemorrhagie myofibroma of the uterus. The galvanism decreased the size of the growth but did not materially lessen the exhaustive hemorrhage. The tumor was of the interstitial variety and 
the uterus appeared the size of a three months' pregnant uterus. When the patient entered the Woman's Hospital for operation December, 1892, she had been having almost continuous hemorrhage for several months. Upon exposing the uterus with the retractors at the time of the operation, the cer vix was large, blue and vascular. As the vagina was large the operation was very easily executed. The ligature on the left side included fully two inches in width of the broad ligament at a distance of at least an inch from the uterus. When I tightened this first ligature one of the spectators, a well known gynecologist, called my attention to the fact that the cervix had perceptibly paled in appearance. The broad ligament was easily exposed on the right side, and fully as much of it ligated as on the left. If there had been any doubt of the procedure affecting the vascularity of the uterus, it vanished when the second ligature was tied. The cervix immediately paled until it was nearly as white as a piece of cartilage.

The covering of the broad ligament was so loosely attached in this case that I could easily feel the main channel of the ovarian artery, and it would have been an easy matter to have included it in the ligature.

After over three years I can promise this case a perfect cure. The uterus has reduced to normal size. The hemorrhage has ceased completely. All pain has disappeared. A slight menstruation, normal in quantity, occurs each month. The patient's health has improved so that from a state of almost complete invalidism she is transformed into to a strong healthy

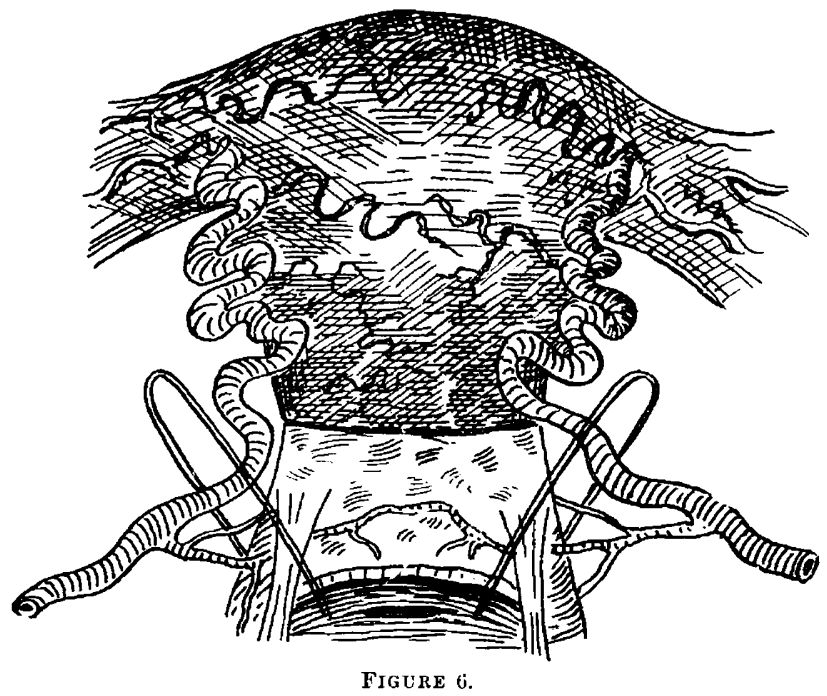

woman. The improvement has been progressive from the day of the operation. I have seen this case within the month (March 1896).

Case 3.-This patient was operated on in January, 1893. She had an incipient interstitial fibroid of two years' standing which was profusely hemorrhagic in nature. I tied the base of both broad ligaments including the uterine arteries and their branches. The relief was immediate. The menstruation for the next four months was scanty. The patient gained in health and strength rapidly. The tumor, which was the size of a four months' pregnancy at the time of the operation decreased markedly in size within three months. Four months after the operation I lost track of this case, as she lived in a distant State and neglected to keep me posted. Her last letter gave a report of perfect health.

Case 4. - This patient had a large, bleeding fibroid filling the pelvis, which extended to the umbilicus. The uterus and appendages were firmly adherent and immovable. Laparotomy had been attempted on the case, with the object of removing the appendages or the tumor. The abdomen was opened, but the adhesions and unusual complications rendered it impossible to remove the tumor or even accomplish the oblation of the appendages. The patient was so unusually reduced from loss of blood at the time of my operation that she had not been able to be out of bed for three months.

I operated on the patient in January, 1893, at the PostGraduate Medical School of Chicago. The operation was accomplished with great difficulty because of the large size and immovability of the uterus. Finally, however, after consum ing more than an hour in time I succeeded in ligating thoroughly the two broad ligaments well above the uterine arteries and their branches.

In June following I made the following report on the case : "The flowing ceased immediately and the patient was relieved "Ther drain for over two weeks. She then had a few days flowing, which resembled an ordinary menstruation. She has rapidly and steadily improved since that time. She has men struated regularly but scantily, and without pain. She can at this time (June, 1893), five months after the operation, attend to her duties as a housewife, and considers herself cured. The tumor has become reduced in size until it is no longer noticeable as a deformity, and so that the patient herself is no longer conscious of its presence.'

Since the foregoing report was written in June, I have seen this patient several times, the last time within the month. The patient was then examined by several physicians, one or two of whom on independent examinations, failed to notice any abnormal enlargement. The uterus is still somewhat larger than normal, but is not more than three or four inches in diameter, while the testimony of at least three experienced diagnosticians will bear me out in the estimate that its former diameters were not less than four and a half by eight inches. The patient is in good health now, Jan. 1, 1895; menstruation is regular but scanty, and she is free from pain. The patient, so far as I know, has remained well.

Case 5. - This case was a woman with an interstitial fibroid about three by five inches. She was about 30 years of age, and the growth had been noticed for three years. Her principle symptoms were profuse menorrhagia with severe menstrual pain. The case was referred to me by Dr. F. H. Greer, of Colum bus, Neb. I did my operation on the woman Jan. 8, 1893. She had a little subsequent temperature, and one month after the operation the ligature sloughed from the left broad ligament. Four months after the operation Dr. Greer reports the woman well. "Menstruation scanty, no pain. Fibroid diminished in size until the uterus is about normal. Patient claims that she is cured." This report was made in June, 1893. I have been unable to get any history subsequent to that date.

Case 6.-This was the wife of a physician of more than ordinary ability and reputation. The patient was about 36 years of age, slightly above the average height, with well-proportioned frame, but poor in flesh, with a skin blanched and a body almost exsanguinated. The uterus was about the size of a three months' gravid uterus. The tumor was uniform and evidently interstitial. The uterus was in normal position. The cervix was nearly two inches in diameter, the os patulous.

The history of the growth dated back, undoubtedly, several years. The patient had borne no children. The menstruation had for nearly two years increased in quantity and duration, until now, while coming with absolute regularity, it lasted fifteen days, and that in spite of vaginal and uterine tampons, the recumbent position, ergot, hydrastis and the rest. She flowed each month until she was completely exhausted, scarcely recovering in the next thirteen days sufficiently so that she could assume the upright position without fain ting. Accompanying this unusual discharge was uterine pain, which in its severity brought the patient to the point of unconsciousness. During the four days in which the woman could drag herself around in the latter part of each intermenstrual period she did so with the greatest discomfort on account of the pressure and neuralgic pains of the pelvis. Upon examination of the broad ligament from the vagina the finger could detect on either side the large, pulsating artery as it fed the tumor. The latter was movable, the appendages apparently normal, the broad ligaments accessible. In fine, here was an ideally typical case-a hemorrhagic fibroid of the uterus, a bed-ridden patient, an authentic diagnosis, an unusually interested physician to carefully watch and estimate the result, and one who enthusiastically demanded a trial of the new operation. Under the circumstances it seemed to me that much depended upon this case, as though the fate of this oper. ation must necessarily be more than usually linked with this particular patient.

I operated on this case Aug. 2, 1894, at the Chicago Hospital. with Dr. Robert Dodds and Dr. Oksschet as assistants. The left broad ligament was carefully dissected from the peritoneal covering behind, and from the bladder in front until fully two-thirds of it could be grasped by placing one finger behind it and another finger or instrument in front of it. When grasped in this manner several beating branches of the uterine 
artery, together with the main artery itself could be detected. This entire mass was then ligated in two sections with No. 12 braided silk, the silk cut short, the parts irrigated and the vaginal wound closed with catgut. After treating the opposite side in the same manner, the vagina was cleansed and loosely packed with iodoform gauze. When the operation was finished the throbbing arteries, which could be distinctly felt before, could no longer be found. The cervix, which was large and purple previous to the operation, became pale and cartilaginous in appearance as soon as the ligaments were secured.

The patient remained in the hospital three weeks. The first menstruation was due the day following the operation. It began the next morning, but was so slight and painless that the patient would not believe that it was her menstruation until several days had elapsed and no other flow appeared. It lasted about three days and was barely perceptible: absolutely no pain. The after treatment consisted in vaginal douches after removing the gauze, light diet and the recumbent position for two weeks.

August 30 , the second menstruation reappeared; there was a little of the old pain, but not sufficient to require anodyne of any kind: the flow was half the usual amount and lasted six days. September 28 , the third menstruation appeared; the amount was normal in quantity, lasting but four days; the pain was slight. October 26 , the fourth menstruation appeared: the amount normal in quantity, lasting but four days: the pain was slight. The patient was seen and examined by me just before the last menstruation. She had gained several pounds in flesh, her cheeks and lips were red and she was a picture of health and robustness. Her feelings were in accord with her appearance, as she enthusiastically assured me that she felt perfectly well. On examination I found the uterus was reduced in size. It was little, if any, larger than normal. Its bulk had decreased one-half. The cervix was small and normal. No arterial pulsation could be felt in either broad ligament or around the vault of the vagina.

The next report I received was in January, 1894: "I have to report," the husband says, " that Mrs. X. menstruated from December 19 to 24 . That the amount was above the same as before, $i$. e., slightly above the normal. Pain rather excessive for two days (possibly due to rheumatism and neuralgia). After flow had ceased I examined and found ligature in vagina and also small sinous opening to left side of cervix. Since then there has been slight discharge from same. She had been suffering some pain at that point, no pain since ligature came away." $\mathrm{He}$ adds enthusiastically: "Taken all in all, the result so far is a grand success." Jan. 17, 1894, he writes: "Mrs. X. is up to-day (the fifth day) after the easiest menstruation she has had in her life: pain moderate and on one day. This in face of the right side still discharging. In the next two months I expect to have a well woman. The uterus is now practically normal."

I have lost track of this patient entirely, and I regret that I am unable to complete so interesting a history. If the husband of this patient should read this report I hope that he will communicate with me.

Case 7.-Mrs. S., Denver, Colo., aged 35, uterus about double the normal proportions, containing two or more centers of development and an extremely hemorrhagic tendency, was the seventh case operated on. The case had been treated unsuc cessfully by curettement, electricity and the ordinary remedies for checking uterine hemorrhages. The uterus was retroverted " but free from adhesions. The patient was prepared carefully, and at the Woman's Hospital, on Nov. 11, 1893, I ligated the base of both broad ligaments, and shortened the round ligi- ments. The uterus was drawn well down, and each broad ligament, after incising the mucous membrane covering them in the vault of the vagina, was dissected free from the bladder and rectal attachments and then ligated with two strong ligatures. These ligatures were placed high enough to include the uterine artery, all its branches, and all of the contents of the base of each broad ligament. The ligatures were cut short after they were tied, the mucous membrane of the vagina was reunited with a running catgut ligature, and the vagina packed with iodoform gauze. The round ligaments were then shortened and the uterus left in a position of anteversion. Three days later the gauze was removed from the vagina, an antiseptic douche was given and a Smith-Hodge pessary was inserted. The antiseptic douches were then continued daily. The first menstruation was due four days after the operation. It did not appear. The second menstruation also failed to appear, notwithstanding the fact that menstruation had ordinarily been exhaustive.

One of the wounds caused in the operation for shortening the round ligament suppurated, and obliged the patient to remain in the hospital until the latter part of January. Dizziness was complained of about the time when the menstruation was due. This symptom continned with different degrees of severity for some time, gradually disappearing. February 13, three months after the operation, the first flow appeared. The patient writes: "First menstruation came on the 13th of this month, without pain, but quite profuse for first two days. Since then has continued, including to-day (the 18th). Discharge light."

March 19, 1894, the patient reports: "Am feeling fairly well this month. Had pain in back with last menstruation. which commenced March 13. First three days quite profuse; last four days very little. No dizziness this month." April 18, she writes: "Menstruation came on four days in advance of schedule time; continued one week. Am in fairly good thealth."

December 14,1895 , two years after the operation, the husband writes that his wife suffers considerably with vertigo, especially severe immediately before menstruation. "The operation performed by you has in a measure been successful, as the menstrual discharge is much less than before the operation and the womb is in much better position."

March 26. 1896, the husband writes: "Her menstruation is not profuse and she has less pain; her general health about the same" (as in her last letter). "I think the riding of the bicycle improves her general health and strengthens her in those parts wherein she is weak."

Case 8. Mrs. Z., Muscatine, Iowa. About 35 years of age. No children. Multiple fibroid of the uterus approximating in size a four months' pregnancy. Hemorrhage prof use, followed for a week by excruciating pain. Patient became extremely exsanguinated at each menstrual period. Frequently the flowing would last for two weeks. The uterus had been curetted. Electricity failed to control the hemorrhage and only partially modified the pain. The irregularity of the uterine canal undoubtedly accounted for the failure of the electricity. Nov. 28. 1893, the patient submitted to my operation for ligation of the broad ligament. The tumor was dereloped more to the left side into the left broad ligament. I succeeded in separating the broad ligament for a height of two inches. On the right side a large double ligature was employed, while on the left side first a double and finally a second one higher and farther away from the uterus was applied. The ligatures were cut short, the vaginal vault closed with catgut and the vagina packed with iodoform drain. The first menstruation was due three days following the operation. A slight watery discharge occurred instead of blood. Two days following the operation the patient complained of pain similar to that which ordinarily occurred after menstruation. Feb. 1, 1894, the patient's husband writes : "She commenced her menstruation January 25, and it was continued until to-day, February 1 , one day less than last time. Had one day of some pain ; not bad. She is getting stronger and can get around the house without being very tired, although 
she has not yet ventured out." February 26 , the report is : "Mrs. Z. was sick this time six days, the same as last time. Had considerable pain two days which was very severe, the same as she complained of before the operation. She is get ting along very nicely. She is now able to go out, and takes a walk every day." March 26, the husband writes: "I am ready to make another report, but not as good a one as I would like. Mrs. Z. was sick on time and the flow was very little compared to what it has been, lasting but three days, but she had a great deal of pain-some before she was sick, and it was quite bad for two days after the menstruation.

Every thing seems to be working very well if she could only get rid of that pain." April, menstruation still decreasing in quan tity ; the pain decreasing. "There was one day of pain" the husband writes, "and the flow amounted to but very little." May 6, he writes: "Mrs. Z . has been feeling splendidly all this last month. Last week was her time to be sick again. The flow did not amount to anything, just enough to show. In regard to her general health, it is excellent. Eats well, sleeps well and goes out every day the same as other women. Has gained her natural amount of flesh and a little more." I examined the patient May 19. The uterus was reduced in size one-half. Patient in perfect health.

March 13, 1896, two years and four months after this woman's operation, I received the following report from this patient: "Since the operation I have gained twenty pounds or a little more up to date. My menstruation period is about one-half the time and amount it was before the operation. The pain is very much less than I had before the operation, but it has not left me altogether. . . . I have the strength of the average woman now, while before the operation I was compelled to be in bed over half the time. Between my menstruations I enjoy as good health as any one could ask.

Case 9.-Mrś. C., aged 41, a resident of Iowa, consulted me for a bleeding painful fibroid of the uterus in May, 1894. The tumor was interstitial, uniform in contour, enlarging the uterus to the size of a four months' pregnancy. 'The hemorrhage at menstruation was profuse and lasted six or eight days at a time. The menstrual periods were accompanied with considerable uterine contractive pains. The patient complained of a great deal of heaviness in the pelvis and pains caused by the pressure of the tumor. The patient was weak, rather exsanguinated and nervous. I concluded that the case was a suitable one for my operation. The operation was done May 19, 1894. The lower portion of the uterus was so large and filled the pelvis so completely that it was with a great deal of difficulty that $I$ accomplished the satisfactory ligation of both broad ligaments. However, when the operation was finished I was well satisfied that both uterine arteries had been thoroughly shut off.

The patient improved from the first. There have been no more hemorrhages. I have examined the patient two or three times since the operation, once within six months. The tumor has decreased in size, but has not disappeared. The pains and pressure symptoms are much better. The woman is apparently a healthy woman and does very much as other healthy women. In reply to my letter of inquiry she said March 12, 1896, one year and six months after her operation: "Since the operation I have had but few hemorrhages, while previous to that I had them very frequently. I am now quite regular, though I never go to my full time-about three weeks. I have less pain, but the heaviness still remains. I am better in health and strength than before the operation."

Judring from my other cases I expect this woman to grailually recover. My fear was, when I adopted this operation, that collateral circulation would speedily overcome the result of ligating of the blood supply. Experience, however, shows that the results of the operation are greater the farther away from the operation we get.

Case $10 .-M r s . S .$, aged 35 , a resident of the central portion of the State, came to the Woman's Hospital in A ugust, 1894, to consult me about an interstitial fibroid. She had borne no children. The uterus was large, regular in contour, hard, and about four by six inches in diameter. It was freely movable in the pelvis. The woman gave a history of severe monthly hemorrhages which lasted anywhere from six diys to two weeks at a time. Accompanying the flooding were severe contraction pains. The woman was bloodless, pale, weak and extremely nervous. In all other respects she was normal.

I did my operation on the case in August, 1894, with the assistance of the house staff of the Woman's Hospital. It was easily performed on account of the movability of the tumor and the looseness of the broad ligaments.

September, 1894, the patient wrote: "It is now six weeks since I have menstruated. The previous pains are not any better. My bladder trouble (pressure) is much relieved." October, 1894 ; "I have menstruated since my last letter. The quantity and length of time was small. Had i good deal of pain the first two days.'

November 13, 1894. "Menstruation three weeks apart. I flowed more than usual."

December 17, 1894. "Pains some less. My changes came at the correct date, but was greater in quantity than it should have been."

January 29,1895 . "I flow a great deal more than I think I ought. I have to change my napkins six or seven times a a day."

March 25, 1895. "I was a little better my sick week this month. The flowing did not last so long as it did before my operation, but more than is right. My pains are gradually improving."

May 27,1895 . "I flowed very freely and had a great deal of pain this month."

Case 11.-Mrs. Y., the wife of a very intelligent physician of Indiana consulted me in November, 1894. She had an interstitial fibroid about the size of a four months' pregnancy. She was 43 years of age. I operated on her Nov. 7,1894 . Both broad ligaments were tied, so as to include two-thirds of their bulk. This occluded the uterine arteries on both sides with all their anomalous branches. I have seen this patient several times since her operation and the uterus is gradually lessening in size and the patient's symptoms are subsiding. I expect this case to prove successful with a little more time. In reply to a request from me for a statement of progress, the husband writes March 12,1894, a year and four months after the opera. tion, as follows: "My Dear Doctor :-In reply to yours of yesterday, I have to say that my wife, on whom you operated Nov. 7,1894 , is doing very well as far as the fibroid is concerned. It has decreased in size some, not a great deal. The menstrual flow on two occasions was quite profuse, but the last two periods have been very scanty only lasting three days, and only using one or two napkins in a day, whereas before the operation she used eight and ten each day for four or five days. She suffers very little pain, in fact none for the last month. Before her operation she suffered constantly. Her general health has greatly improved, and she has gained ten pounds in flesh, is much more cheerful, and in fact improved in every way.

My wife is now past 45 years of age, and I believe if the tumor does not increase in size until after the menopause, she will entirely recover."

Case 1:.-Miss V., single, age 40, consulted me in November, 1894 , on account of a painful bleeding fibroid. She was depleted to an unusual state and her nervous system was a wreck. She had an interstitial fibroid with the canal of the uterus measuring four inches in depth. The uterus measured approximatelv three by six inches in diameter. The organ was movable. Hemorrhage occurred only at regular menstruation periods. At this time it lasted a week or ten days and was very profuse. Accompanying the flow was great prostration of the patient and also most excruciating pelvic pressure symptoms. This condition of affairs had been going on for months until the patient from blood drain and harassment of pain had been brought to a deplorable state of health.

March 5, 1894. I ligated the base of both broad ligaments. The operation was accomplished with ease because the uterus was movable and the broad ligaments loose. The patient recovered nicely from the operation. The next two or three menstruations were much more normal, the quantity of flow being very small and the pain scarcely perceptible. The woman was placed on tonics and urged in every way to increase her blood supply. Her nervous system reacted slowly. Her menstruations later became more profuse and was accompanied on several occasions by quite severe pain. While but a short time has elapsed since the operation the patient is gradually improving.

March 12, 1896, one year and three months after the operation, she writes: "The amount of menstrual flow averages 
about one-half the amount it was before the operation. Have gained a little in flesh. Have considerable pain. Still have nerves although under better control than formerly."

This patient has improved in many ways.

During her intra-menstrual periods she is comparatively well and is able to go about and to do more work than she should attempt. whereas previous to her operation she was unable to do much. While she had been neglected for a long time and her health had reached a low ebb. I can not but believe that she will gradually improve as a direct result of the diminished flow. Her tumor decreased in the first four weeks fully one-third. It has not increased perceptibly to the patient since she left my care.

Case 13.-Miss B., age 26, consulted me in January, 1895, for a bleeding intramural fibroid. The uterus was about five inches long and had a canal three and three-quarter inches in depth. The canal was a little irregular. In the fundus of the uterus could be felt two distinct centers of development, one on the interior surface about two and one-half inches in diameter, and projecting from the main body of the uterus one and one-half inches. It was hard and had the unmistakable firm consistency of a fibroid mass. On the posterior surface of the fundus at its junction with the neck was a second center projecting from the uterus about one and a half inches. This mass was irregular and was fully two inches in diameter. The symptoms which brought this patient to me were prolonged and exhaustive hemorrhages and uterine pains. The lady is a vocalist of unusual talent and these symptoms interfered seriously with her profession. The case was a typical one for hys terectomy, especially as the left ovary was enlarged and cystic, but as that would involve the removal of the ovaries the patient objected to this because of the popular but unfounded fear that removal of the ovaries impairs the voice. I therefore decided to perform my operation on the case. When the patient was under the anesthetic I confirmed absolutely by bimanual manipulation my diagnosis as given above. February 1, 1895, I operated on this patient. She left the hospital in two weeks. She did not have an unfavorable symptom. Menstruation practically ceased from the date of the operation. There was but the slightest show each month. No pain whatever. In less than a month she was able to attend to her proressional duties and was stronger than she had ever been. This perfect condition of affairs continued until about Dec. 8, 1895, ten months after her operation. At this time I was called because of a sudden attack of severe pain she had experienced in the left side of the pelvic region. The pain was accompanied with profound prostration and shock. I diagnosed ruptured cyst of the left side and advised a laparotomy. In making my examination I was surprised to find a perfectly normal uterus. I performed laparotomy on this patient Dec. 28, 1895, and removed a ruptured ovarian cyst of the left side and punctured a small cyst in the ovary of the right side. This gave me an opportunity to examine the uterus which I had treated by my operation a little over ten months before. On the anterior portion of the organ corresponding to the location of the anterio fibroid described above $I$ found buried in the wall and project ing a half inch, a fibroid center one-half inch in diameter. On the posterior surface, corresponding to the other center which I palpated at the previous operation, was another center dis tinct but even smaller than the anterior one. These were both exhibited to the house staff and physicians present at the oper ation. The behavior of this case was most gratifying until the complication of the ruptured cyst arose. This fortunately gave me an opportunity of examining by direct sight the results accomplished by the first operation.

I have no doubt but that those two fibroid centers would have been starved out eventually and the case actually cured without any further interference.

General Summary: Thirteen cases operated on in which more than a year has elapsed since the operation:

Case 1.--Age 40. Operation Nov. 15, 1892. Very large bleeding fibroid. Present condition: Tumor much reduced. Hemorrhages ceased. Patient well.

Case 2.-Age 40. Operation December, 1892. Fibroid interstitial, size of three months' pregnancy. Profusely hemorrhagic. Present condition: Tumor disappeared. Absolute cure.
Case 3.-Operation January, 1893. Interstitial bleeding fibroid of two years standing. Four months after operation. Last report: Tumor reduced, patient much improved.

Case 4. Age 38. Operation January, 1893. Very large adherent interstitial fibroid. Excessively hemorrhagic. Patient bed-ridden. Two years afterward : Uterus reduced almost to normal size. Hemorrhage ceased. Patient well and strong. Case 5.-Age 30. Operation Jan. 8, 1893. Interstitial fibroid three by five inches in diameter. Profuse hemorrhage. Report four months after operation: Uterus normal; hemorrhage ceased.

Case 6.-Age 36. Operation Aug. 2, 1894. Interstitial fibroid. Profusely hemorrhagic and painful. Patient much reduced. Tumor three by five inches in diameter. Six months: after operation much improved. No later report.

Case 7.-Age 35. Operation Nov. 11, 1893. Incipient interstitial bleeding fibroid. Two years after operation: Tumor reduced; hemorrhage ceased.

Case 8.- Age 35. Operation Nov. 28, 1893. Painful, hemorrhagic interstitial fibroid, size of four months' pregnancy. Two years and four months after operation: Tumor much dimished; hemorrhage ceased : pain less but not entirely relieved. Case 9. Operation May 19, 1894. Interstitial, painful, hemorrhagic fibroid. Tumor size of four months' pregnancy. One year and ten months after operation: Tumor slightly diminished; hemorrhage materially reduced.

Case 10.-Age 35. Operation August 1894. Tumor interstitial four by six inches in diameter. Hemorrhage and pain excessive. Not much improved eight months after operation.

Case 11.- Operation November 7, 1894. Tumor interstitial, hemorrhagic, painful and size of four months pregnancy. One year and four months after operation: Tumor decreased in size and hemorrhage ceased.

Case 1\%.-Operation Nov, 1894. Tumor interstitial, profusely hemorrhagic, painful, and three by six inches in diameter. One year and three months after operation: Tumor reduced; hemorrhage less; pain not improved.

Case 13.-Operation Feb. 1, 1895. Tumor intramural, two centers of development two inches in diameter each. Profusely hemorrhagic and excessively painful; hemorrhage and pain ceased ; tumor disappeared, as demonstrated by a laparotomy ten months later.

MINOR SURGERY FOR SUBMUCOUS FIBROIDS.

Pedunculated submucous fibroids may frequently be completely removed through the dilated cervix without interfering materially with the uterus. Unless the tendency to pedunculate is well established however, and the center of development comprising the tumor is the only center of fibroid development to be discovered in the walls of the uterus as shown by careful bimanual palpation, it should be treated by hysterectomy either vaginal or abdominal. An exception to this general rule would be when a pedunculated fibroid is discoverable either in the cavity of the uterus or hanging from the cervix with a long thin pedicle. In such a case the polypus should be care. fully removed from the uterus, even though there were other centers of development to be discovered. The uterus as a whole, here, could be dealt with in a later operation if the removal of the pedunculated mass did not sufficiently relieve the symptoms.

The removal of an intrauterine pedunculated fibroid is usually a simple procedure. If the pedicle is small and long and the tumor is in a position where it can be easily reached with forceps, it may be grasped in a strong vulsellum and the tumor twisted until the pedicle is actually twisted in two. This can only be done with thin pedicles. If the pedicle is broad the uterus should be sufficiently dilated (the patient under an anesthetic) to expose the pedicle, if it is necessary to accomplish this the cervix may be divided as high as the vaginal junction. The mucous membrane of the pedicle should next be cut in its entire circumference. Then the remaining portion of the pedicle composed of the blood vessels, connective and muscular tissue should be twisted in the same way that one proceeds to twist off a small pedicle. If 
the remaining portion of the pedicle is small it will give way by that treatment. If it is rather large and fleshy, after it has been twisted into a small bulk it may be grasped by a strong pair of curved pedicle forceps and the pedicle severed with scissors or a knife outside of the forceps. If the pedicle is very vascular the forceps may be left in place for six or twelve hours. If this does not seem necessary the forceps are removed and the uterus packed with iodoform gauze. If the forceps are left on the pedicle, gauze should be packed around them. The forceps may be removed in six or twelve hours without disturbing the gauze.

I do not favor attempting to enucleate a submucous fibroid of any considerable size if its principal bulk is buried in the walls of the uterus. Such a procedure is attended with considerable mechanical difficulty because of the position of the tumor in the cavity of the uterus; it is a difficult matter to secure hemostasis in such a location and finally one seldom reaches in such a procedure more than one of several centers of developments of the tumors which are situated in the uterus. In these cases a hysterectomy is more satisfactory.

A cervical fibroid developing toward the mucous membrane, if pedunculated, should be removed in the same manner as that described for removing a pedunculated intrauterine fibroid. A cervical fibroid of small size may be enucleated by incising its capsule, yrasping the tumor with a vulsellum, and dissecting it from its bed. The cavity may be closed with buried antiseptic catgut sutures or it may be packed with iodoform gauze.

\section{C'URETTEMENT}

In many cases of hemorrhayic fibroids much of the hemorrhage and leucorrhea is caused by endometritis. A safe and oftentimes beneficial treatment for such cases is thorough dilatation of the uterine canal and curettement of its mucous membrane. While it will not ordinarily have a direct curative effect it will frequently relieve disagreeable symptoms for a long period of time. The dilatation should be gradual beginning with Goodell's dilators and afterward exploring the anterior of the uterus with the index finger to discover whether there are any projecting masses into the interior of the cavity. After thorough dilatation with the cervix exposed and grasped with small vulsellum forceps in order to steady the whole organ, a sharp curette should be made to traverse all portions of the endometrium. This should be accompanied with some form of antiseptic irrigation. The whole mucous membrane should be gone over at least three times with the curette, the canal then loosely packed with iodoform gauze, the vagina filled with the same and the patient put to bed for several days. The gauze should be removed in fortyeight hours. After that antiseptic vaginal 'douches must be given for several days.

The Latin Part of the Body,-Medico Lecks: The deceased was shot between the hyoid bone and the insertion of the stermo-cleido-mastoid muscle.

District Attorney Rockaway: Do I understand you to say that wounds in this Latin part of the body are generally fatal?
TWO EXPERIMENTS IN RESTORING LOST SPEECH, OR PARTIAL RESTORATION OF SPEECH BY SYSTEMATIC EDUCATION.

BY L. PIERCE CLARK, M.D. CRAIG COLONY, SONYEA, N. Y.

While reading one of Professor Sully's articles on Childhood, the thought occurred to me that, in many cases of aphasia in people suffering from apoplexy of the brain and also in the allied disorders of softening and actual breaking down of different portions of the brain, there existed a striking analogy between such attempting to acquire the language anew and the child's attempts in learning to speak for the first time.

I recall two cases that give interesting and peculiar illustrations of this analogy. The first, a subject of right side paralysis, had been unable to speak at all, with the exception of the words, "gully" and "damn." The former she uses at all times and the latter only at periods when she becomes very much excited. This word "gully" she repeats three or four times in varying degrees of tone and pitch, as though each repetition of the word had a distinct and separate meaning and the whole represented some phrase or thought as in normal expression of ideas. The word "gully" is generally accompanied by a knowing look, a gesture of the hand, elevation of the eyebrows and tossing of the head from side to side. These acces. sories of gesture and modulations of voice are evidently very necessary to herself for proper expression, because when the hand and head movements are interfered with, she becomes very angry and will take no notice of anyone speaking to her, but when released will chatter on in her one word vocabulary, not at all reluctant to engage in conversation with any one upon any subject. When she becomes extremely angry, which she does upon slight provocation, she explosively emits the single word "damn" and covers her face with her hands in apparent shame because of such exhibition of anger. She readily understands most things said to her, as indicated by her quick compliance with one's commands. Her paralysis hinders her from taking an active part in any heavy work, but she is able to keep her own immediate surroundings in perfect order and cleanliness. Of late she has become quite fond of her nurse and, strange to relate, by the persistent endeavor and painstaking care of her instructress, the patient has been induced to copy the word sounds as given to her by her nurse. She keeps close watch of the nurse's lip movements and imitates them closely. It is needless to say that her progress in regaining the language has been $\mathrm{ex}^{-}$ ceedingly slow and only by the exhibition on the part of the nurse of those cardinal virtues of the true teacher, patience and tact, has she been able to keep the patient interested. The unfortunate woman can now make known some of her wants and desires quite intelligently, such as "I want a drink of water," and "Let me go out," to say nothing of the disconnected ideas as expressed by single and salient words combined with the appropriate sign language. By this peculiar method, saying "soap-water-towel," and again "crutches-walk-door-air," these phrases are quite readily interpreted into sentences expressing the desire that she wishes to have soap, water and towel, in order that she may cleanse her face and hands, and that she would like her crutches in order 